\title{
Modalidades de atención y desigualdad educativa en tiempos de pandemia: la experiencia de la Sierra Tarahumara
}

\author{
Jaime César Mendoza Caro (D); Jordi Abellán Fernández $\mathbb{1}$ \\ Universidad Pedagógica Nacional del Estado de Chihuahua (UPNECH), México
}

Resumen. El propósito de este artículo es documentar las experiencias que enfrentan un grupo de docentes de educación básica en la Sierra Tarahumara del Estado de Chihuahua, México, ante la contingencia sanitaria provocada por la COVID-19. La recolección de datos se realizó a través de un cuestionario electrónico de respuesta múltiple y una entrevista semiestructurada para determinar si las estrategias remediales promovidas por la Secretaría de Educación Pública responden a las características de una región caracterizada por la marginalidad, la precariedad tecnológica y los problemas de comunicación. En el estudio se emplea una metodología mixta con base en la técnica narrativa, que se complementa con una descripción de frecuencias absolutas y porcentajes conectados con las dificultades que comparten los maestros. Los resultados de la investigación revelan una intensificación en las actividades diarias, un notable compromiso con la profesión y ponen al descubierto los desafíos que confronta el profesorado para contener las pérdidas de aprendizaje. Asimismo, los hallazgos más críticos se pueden utilizar como insumo para el diseño de medidas de política pública educativa encaminadas a la atención inclusiva de los estudiantes en riesgo de fracaso escolar.

Palabras clave: derecho a la educación; política educativa; educación a distancia; desigualdad educativa; COVID-19.

Modalidades de atendimento e desigualdade educacional em tempos de pandemia: a experiência da Serra Tarahumara

Resumo. O objetivo deste artigo é documentar as experiências enfrentadas por um grupo de professores de educação básica na Serra Tarahumara, no Estado de Chihuahua, México, diante da contingência sanitária causada pela COVID-19. A coleta de dados foi realizada através de um questionário eletrônico de múltipla escolha e uma entrevista semiestruturada para determinar se as estratégias de remediação promovidas pela Secretaria de Educação Pública respondem às características de uma região marcada pela marginalidade, precariedade tecnológica e problemas de comunicação. O estudo utiliza uma metodologia mista baseada na técnica narrativa, que se complementa com uma descrição de frequências absolutas e porcentagens ligadas às dificuldades compartilhadas pelos professores. Os resultados da pesquisa demonstram uma intensificação das atividades diárias, um compromisso notável com a profissão e revelam os desafios que os professores enfrentam para conter a perda de aprendizagem. Além disso, os resultados mais críticos podem ser utilizados como insumo para a elaboração de medidas de políticas públicas educacionais encaminhadas ao cuidado inclusivo de estudantes em risco de fracasso escolar.

Palavras-chave: direito à educação; política educacional; educação à distância; desigualdade educacional; COVID-19.

\begin{abstract}
Modalities of care and educational inequality in pandemic times: the Sierra Tarahumara experience

Abstract. The purpose of this paper is to document the experiences faced by a group of basic education teachers in the Sierra Tarahumara in the State of Chihuahua, Mexico facing the health contingency caused by COVID-19. Data collection was carried out through a multiple response electronic questionnaire and a semi-structured interview to determine if the remedial strategies promoted by the Ministry of Public Education respond to the characteristics of a region distinguished by marginality, technological precariousness and communication problems. This study uses a mixed methodology based on the narrative technique, which is complemented with a description of absolute frequencies and percentages connected with the difficulties shared by the teachers. The research results reveal intensification in daily activities, a remarkable commitment to the profession and also reveals the challenges that teachers face in containing learning losses. Furthermore, the most critical findings can be used as input for the design of educational public policy measures aimed at inclusive care of students at risk of school failure.
\end{abstract} Keywords: right to education; educational policy; distance education; educational inequality; COVID-19. 


\section{Introducción}

La pandemia de la COVID-19 ha modificado el trabajo docente, la organización de las escuelas y los sistemas educativos, aunque las principales consecuencias se observan en la desigualdad de oportunidades para el aprendizaje. En un informe elaborado por el Banco Mundial (2018) se dejaba constancia de la crisis de aprendizaje que ya entonces afrontaba el mundo: "cientos de millones de personas llegan a la primera etapa de la adultez sin contar siquiera con las competencias más básicas para desenvolverse en la vida" (p. 3). El concepto "pobreza de aprendizajes", creado por este organismo, se refiere a los alumnos que no han logrado adquirir un nivel mínimo en lectura e incluye al $53 \%$ de los niños de países de ingreso bajo y mediano: 9\% privados de escolarización y 44\% sin opciones de aprender (Banco Mundial, 2019), una cifra que se estima va a ascender al 63\% después de la pandemia (Banco Mundial, 2020). En este escenario, la suspensión de clases corre el riesgo de que se traduzca en tiempo sin aprendizaje y aumento en la deserción, una brecha en términos de inclusión, equidad y calidad que afecta primordialmente a los niños, niñas y jóvenes que pertenecen a grupos vulnerables. En este tenor, el artículo pone a discusión si la educación a distancia asegura el mismo aprendizaje para todos los alumnos y por eso, los resultados que se presentan y la evidencia similar deben integrarse a la agenda de investigación sobre la deprivación sociocultural en las siguientes décadas.

Los diagnósticos objetivos pueden parecer desalentadores, pero dejan de serlo cuando las autoridades asumen la divisa de que es posible el derecho a la educación para los estudiantes más desfavorecidos. El cuarto Objetivo de Desarrollo Sostenible de la Agenda 2030 indica que es necesario "garantizar una educación inclusiva y equitativa de calidad y promover oportunidades de aprendizaje permanente para todos" (UNESCO, 2017, p. 8). Al respecto, se resalta el compromiso de cuidar "el acceso en condiciones de igualdad de las personas vulnerables, incluidas las personas con discapacidad, los pueblos indígenas y los niños en situaciones de vulnerabilidad" ( $p$. 7). Por esta causa, ninguna meta educativa se puede considerar alcanzada si no abarca a todos los estudiantes.

Más allá de la omisión a la que se ven sometidos algunos sectores de la población cuando se manejan estadísticas nacionales o regionales, el efecto de la pandemia en los niveles de aprendizaje queda patente si tomamos en cuenta el origen social y étnico, en la que destacan los alumnos de poblaciones rurales e indígenas. Aunque la exclusión también se produce cuando la evaluación está diseñada con el fin de encubrir la realidad educativa, tanto en circunstancias normales como en las actuales. En concreto, las diferencias se difuminan con la complicidad de todos los actores sociales y el fracaso, el rezago o la discontinuidad en el aprendizaje se disimulan bajo el efecto adormecedor de los discursos optimistas, las calificaciones aprobatorias y la promoción.

\section{Marco educativo de la pandemia}

En marzo de 2020 cerraron los centros escolares de más de 190 países del mundo con el propósito de evitar la transmisión del virus y aminorar su impacto, con lo que cerca de 1200 millones de estudiantes dejaron de tener clases presenciales, más de 160 millones en América Latina y el Caribe (CEPAL-UNESCO, 2020). En 
enero de 2021 todavía aprendían desde sus casas 681 millones de alumnos (Banco Mundial, 2021). En esta coyuntura, las familias se vieron obligadas a elegir entre el aprendizaje remoto o la desescolarización. En México y en la mayoría de los países de Latinoamérica, las autoridades decidieron recurrir a modalidades improvisadas para dar continuidad al servicio escolar. Las medidas que se adoptaron fueron diversas, unas de carácter prescriptivo y otras producto de la capacidad de adaptación de los docentes, aunque todas se deberían valorar desde su idoneidad para construir aprendizajes y no solo para mantener el vínculo entre la escuela, los estudiantes y sus familias.

En América Latina y el Caribe se implementaron programas de emergencia para gestionar el aprendizaje a distancia y el servicio educativo a través de recursos en línea. En 29 países de la región las estrategias que la IIPE-UNESCO (2020) contabilizó fueron el uso de instrumentos de aprendizaje (29), aprendizaje en línea (26), aprendizaje sin conexión a Internet (24), transmisión de programas educativos (por televisión o radio) (23) y plataforma en línea de aprendizaje a distancia (18). Más que una respuesta basada en una planeación previa se trató de procesos de ensayo y error que sufrieron ajustes sobre la marcha, tanto en el calendario escolar, como en la adaptación del currículum y la enseñanza por medios digitales:

Los países que contaban con plataformas virtuales de contenidos educativos pusieron el foco en su adecuación y actualización. Los demás países pusieron en línea nuevas plataformas virtuales, en algunos casos en cooperación con empresas como Microsoft, Cisco y Google, y con organismos multilaterales. En la mayoría de los casos, las plataformas de contenido se complementaron con soluciones de aulas virtuales (CEPAL-ONU, 2020, p. 9).

Las dos prioridades en política pública de los países de la región buscaban asegurar el contacto con "aquellas poblaciones que tienen mayores dificultades de conexión y se encuentran en condiciones sociales y económicas más desfavorables para mantener procesos educativos en el hogar" (CEPAL-UNESCO, 2020, p. 4) y, en "proyectar procesos de recuperación y continuidad educativa para el momento de reapertura de las escuelas, que consideren las diferencias y las desigualdades que se profundizarán en este período" (p. 4).

En México, el gobierno federal creó una propuesta denominada "Aprende en Casa" para difundir temas educativos por varios canales de televisión, una estrategia que contiene cápsulas por nivel y grado de educación básica, y que en esencia incluye actividades extraídas de los libros de texto (Navarrete, Manzanilla y Ocaña, 2020). También se recurrió a emisoras de radio para transmitir el programa "Aprendiendo desde mi comunidad" con contenidos en quince lenguas originarias (Baptista, Almazán, Loeza, López y Cárdenas, 2020), se diseñaron cuadernos de trabajo para los alumnos en situación de vulnerabilidad, se emplearon los libros de texto gratuitos y colecciones de fichas editadas por la Secretaría de Educación Pública (SEP). A su vez, la asesoría pedagógica de los maestros por medio de las redes sociales (Facebook, WhatsApp, etc.) o de plataformas como Zoom, Meet o Classroom han sido los procedimientos más recurrentes desde que inició la pandemia.

Estos arreglos didácticos intentan solventar problemas como el abandono escolar, los bajos niveles de aprovechamiento, la escasa disponibilidad de tecnología y conectividad o la disparidad que hay entre diferentes regiones del país. No obstante, 
en México y otros estados de Latinoamérica la permanencia del servicio educativo se relaciona más con la necesidad de justificar el salario de miles de maestros que con el aprendizaje de los alumnos. Por añadidura, la educación a distancia ha demeritado la función docente y de ser una actividad formativa se ha convertido en un remedio para no detener la maquinaria escolar, económica y social.

\section{Método y materiales}

La Sierra Tarahumara, situada en la franja suroeste del Estado de Chihuahua, se caracteriza por la combinación de zonas montañosas y barrancas, tiene una extensión de 59874 km², agrupa a 24 municipios y su densidad es de 5.4 habitantes por km². Los 302066 mestizos e indígenas rarámuri, guarijó, o'ob y o'dam que viven en la región corresponden al $8.1 \%$ de la población estatal. Chihuahua ocupa el décimo lugar a nivel nacional en el índice de marginación, aunque 15 municipios de la Sierra están en condición de muy alta o alta marginación, integran a 218933 personas y representan el $72.5 \%$ de la población (INEGI, 2021).

Tabla 1. Datos demográficos de la Sierra Tarahumara, Chihuahua

\begin{tabular}{lrcccc}
\hline Municipios & Población & Densidad & $\begin{array}{c}\text { Población } \\
\text { activa (\%) }\end{array}$ & $\begin{array}{c}\text { Hablantes } \\
\text { indígenas (\%) }\end{array}$ & $\begin{array}{c}\text { Grado de } \\
\text { marginación }\end{array}$ \\
\hline Batopilas & 11270 & 5.3 & 37.5 & 59.0 & Muy alto \\
Carichí & 8113 & 3.1 & 39.2 & 49.5 & Muy alto \\
Urique & 17043 & 5.2 & 37.4 & 48.6 & Muy alto \\
\hline Balleza & 16440 & 3.1 & 39.4 & 49.6 & Muy alto \\
\hline Guachochi & 50180 & 7.2 & 69.6 & 56.1 & Muy alto \\
\hline Guadalupe & 50514 & 5.3 & 62.8 & 33.9 & Muy alto \\
\hline Morelos & 7266 & 3.3 & 45.6 & 32.6 & Muy alto \\
\hline Maguarichi & 1302 & 1.3 & 36.5 & 16.8 & Muy alto \\
\hline Uruachi & 6512 & 2.4 & 25.6 & 21.0 & Muy alto \\
\hline Guazapares & 8196 & 4.5 & 53.9 & 26.0 & Alto \\
\hline Bocoyna & 23351 & 8.6 & 61.3 & 14.6 & Alto \\
\hline Chínipas & 6222 & 3.1 & 50.0 & 13.6 & Alto \\
\hline Temósachi & 5320 & 1.2 & 48.6 & 6.6 & Alto \\
\hline Moris & 4447 & 2.5 & 48.6 & 3.8 & Alto \\
\hline Nonoava & 2757 & 1.4 & 46.0 & 13.9 & Alto \\
\hline
\end{tabular}

Fuente: elaboración propia a partir de INEGI (2021) y COESPO (2017).

En la Tabla 1 resaltan los altos porcentajes de desempleo, la dispersión geográfica y el reducido número de habitantes, así como la correspondencia que hay entre el grado de marginación y los hablantes de una lengua indígena. Estos rasgos se combinan en una región con una dinámica socioeconómica marcada por la producción de enervantes, la violencia, la migración, el turismo y la explotación indiscriminada de los recursos forestales y mineros.

La investigación no remite a un corpus teórico de referencia porque se aborda un tema emergente que en un futuro va a implicar un tratamiento conceptual específico. En síntesis, se trata de un trabajo exploratorio basado en evidencia empírica y de corte 
descriptivo-explicativo que pretende avanzar en el conocimiento de las modalidades de atención relacionadas con la educación a distancia y el impacto de la pandemia sobre los niveles de aprendizaje.

El estudio corresponde a un diseño mixto de tipo cuantitativo-cualitativo (Johnson y Onwuegbuzie, 2004) que se desarrolló durante los ciclos escolares 2020-2021 y el primer trimestre del 2021-2022. En la investigación participaron 37 maestras y docentes de educación preescolar, 68 de primaria y 28 de secundaria repartidos en 11 municipios de la Sierra Tarahumara que reúnen las siguientes características educativas.

Tabla 2. Datos de educación básica. Sierra Tarahumara, Chihuahua

\begin{tabular}{lccccc}
\hline \multicolumn{1}{c}{ Municipios } & Escuelas & Maestros & Alumnos & $\begin{array}{c}\text { Asistencia } \\
\text { escolar (\%) }\end{array}$ & Alfabetización (\%) \\
\hline Batopilas & 132 & 223 & 3628 & 64.3 & 63.1 \\
\hline Carichí & 51 & 110 & 1764 & 60.7 & 65.3 \\
\hline Urique & 143 & 321 & 5885 & 80.7 & 97.9 \\
Guachochi & 380 & 836 & 14701 & 84.4 & 91.2 \\
Guadalupe & 509 & 830 & 13425 & 83.5 & 89.4 \\
Morelos & 99 & 144 & 2252 & 83.1 & 86.6 \\
Maguarichi & 10 & 22 & 425 & 87.9 & 95.2 \\
\hline Uruachi & 106 & 156 & 1999 & 86.8 & 94.0 \\
\hline Guazapares & 75 & 175 & 2427 & 87.7 & 93.2 \\
Bocoyna & 196 & 541 & 8395 & 90.4 & 97.4 \\
Chínipas & 72 & 164 & 2369 & 93.6 & 96.7 \\
\hline
\end{tabular}

Fuente: elaboración propia a partir de INEGI (2021) e INEGI y SEP (2014).

El cruce entre el número de maestros y escuelas de la Tabla 2 da una razón de 2.03, con tres municipios que se sitúan por debajo de la media. Ala par, la proporción de 16 alumnos por docente corrobora los datos estatales sobre escuelas multigrado: 332 preescolares ( $78.7 \%$ del subsistema indígena), 800 primarias $(77.3 \%$ del subsistema indígena) y 193 telesecundarias unitarias o bidocentes (INEE, 2019). Por último, el bajo porcentaje de asistencia escolar y el grado de analfabetismo anticipan el acompañamiento escolar que pueden ofrecer algunas familias durante la educación a distancia.

La edad promedio de las 110 mujeres y 23 hombres incluidos en el estudio es de 30.8 años, el 52\% tienen de 1 a 5 años de servicio, el 30\% de 6 a 10 años y el 18\%, 11 o más años. En la parte cuantitativa se empleó un muestreo por disponibilidad y se estableció comunicación con los participantes mediante la aplicación de WhatsApp gracias a la colaboración de 10 supervisores estatales y federales que proporcionaron los datos de contacto. En la investigación cualitativa los informantes clave se eligieron por modalidad educativa tomando en cuenta que el $73 \%$ trabajan en escuelas multigrado, el $27 \%$ en escuelas de organización completa y del total, el $22 \%$ en escuelas indígenas.

Para la recolección de datos se utilizó un cuestionario electrónico que se envió a 250 maestros y directores frente a grupo, contestado en línea por 133 docentes a través de Google Forms. En las instrucciones se agradeció su cooperación, se clarificó que era confidencial y tenía como objetivo conocer diferentes realidades educativas 
ante la pandemia. El instrumento está conformado por siete categorías que agrupan 25 reactivos con 4, 5 o 6 opciones de respuesta: las primeras cinco preguntas reúnen información acerca de la antigüedad en el servicio, la función que cumplen, el sistema, el grado, el tipo de organización, el nivel en que enseñan y el lugar donde se localiza la escuela; las siguientes cuatro indagan sobre los servicios públicos con que cuentan y los recursos para atender a los estudiantes; ocho más tratan de los procesos de planeación, comunicación, acompañamiento y evaluación del aprendizaje; enseguida, se incorporan dos preguntas sobre la implementación de las estrategias que propone la SEP y la capacitación que les gustaría recibir; por último, se solicita que realicen una valoración de su quehacer y se cuestiona si la educación a distancia les ha provocado o no estrés laboral. El análisis cuantitativo se hizo a partir de las secciones en que se ordena el cuestionario y recurre a frecuencias absolutas y porcentajes.

La metodología cualitativa se desarrolla con base en una entrevista semiestructurada que se efectuó por vía telefónica a 12 maestros para profundizar en sus experiencias y se reconoce en el texto con un código que indica el número de entrevista $(\mathrm{E} 1)$, el nivel educativo ( $\mathrm{PR}=$ preescolar; $\mathrm{P}=$ primaria; $\mathrm{S}=$ secundaria, $\mathrm{TS}=$ telesecundaria), el tipo de organización ( $\mathrm{C}=$ completa; $\mathrm{M}=$ multigrado; $\mathrm{TS}=$ telesecundaria) y de alumnado ( $\mathrm{I}=$ indígena; $\mathrm{G}$ = general). Los informantes proporcionaron datos socioeconómicos y laborales, explicaron su percepción en torno a las medidas propuestas por la SEP para atender las consecuencias de la pandemia, ofrecieron alternativas para mejorar el logro educativo, precisaron qué retroalimentación entregan a los alumnos y las carencias que detectaron en los padres de familia para ayudar a sus hijos, detallaron las acciones que realizan durante el proceso de preparación y evaluación de la enseñanza, y los aspectos que más les preocupan de la educación a distancia.

El proceso de descripción y análisis se divide en cinco segmentos: servicios básicos y recursos educativos, atención a los estudiantes, funciones docentes, formación continua, desempeño y deterioro profesional. En cada apartado se incorporan las principales características que arrojan los instrumentos, luego se comparan las tendencias que prevalecen en docentes y estudiantes, se añaden testimonios que ahondan en cada hecho o suceso y los resultados se contrastan con estudios nacionales e internacionales sobre la atención a la emergencia sanitaria y educativa. El diseño mixto corresponde a la estrategia concurrente de triangulación que formula Creswell (2003) porque se busca fortalecer los supuestos del conocimiento y explicar las ausencias de convergencia que pudieran resultar, para obtener así una mayor comprensión del objeto de estudio.

\section{Situación de desigualdad educativa}

Verbigracia. Una docente de preescolar resume en pocas palabras el panorama de la educación en tiempos de pandemia: "El sistema educativo en México está pensado para un espacio físico. No estamos preparados para la educación a distancia: las diferencias sociales no lo permiten" (E5-PRMI). Las mismas autoridades reconocen que en estos momentos la atención educativa hace frente a circunstancias especiales:

que se expresan en desigualdad de condiciones materiales para el estudio en casa, tales como el acceso a dispositivos tecnológicos, la conectividad y la disposición de espacios adecuados en el hogar. Asimismo, influyen en esos 
niveles de vinculación factores de otro orden, como el tiempo disponible, las relaciones intrafamiliares, el interés o disposición de los adultos a acompañar a sus hijas, hijos o pupilos en el estudio o el nivel de comprensión que poseen sobre los contenidos escolares (Acuerdo número 26/12/20, pp. 1-2).

Por consiguiente, como afirma la SEP (2021), "sería injusto atribuir a la irresponsabilidad o falta de interés de los alumnos un bajo nivel de involucramiento, o el escaso avance en su aprendizaje, cuando enfrentan barreras de aprendizaje" (p. 2). Dicho de manera clara, importa mantener en funcionamiento al aparato administrativo y no tanto que se frene o retrase el proceso de aprendizaje.

\subsection{Servicios básicos y recursos educativos}

La existencia de la escuela en tiempos de pandemia solo se justifica desde las posibilidades del alumnado para participar en las actividades que se desarrollan a distancia. La SEP plantea tres niveles de comunicación y participación en los estudiantes de educación básica: sostenida, cuando se ha dado seguimiento a la programación de "Aprende en casa" y la comunicación ha sido constante entre el maestro y los alumnos, han intervenido en las sesiones programadas y resuelto las actividades didácticas; intermitente, cuando el seguimiento de "Aprende en casa" ha sido ocasional, la comunicación se ha producido de forma esporádica y se han entregado parcialmente los trabajos; e inexistente, cuando no ha habido posibilidad de dar seguimiento al proceso educativo de los alumnos (Acuerdo número 26/12/20). Aparte de la cantidad de estudiantes que queden repartidos en cada categoría, no debemos obviar que ser espectador pasivo de un programa de televisión, entregar tareas y estar en comunicación no son suficientes para construir aprendizajes.

El hecho de que la educación haya migrado al ámbito digital más que contener el impacto de la pandemia ha dejado al descubierto la disparidad de acceso a los dispositivos electrónicos y a Internet. Un estudio dirigido por la CEPAL-ONU (2020) aseguraba que "el $46 \%$ de los niños de entre 5 y 12 años de la región viven en hogares que no están conectados a Internet" (p. 9), aunque en México la conectividad disminuye al $11 \%$ y $28 \%$ para los dos quintiles más bajos de la distribución del ingreso. Por otro lado, los estudiantes de 15 años que contaban en 2018 con dispositivos digitales apenas alcanzaban el $12 \%$ en el cuartil socioeconómico más bajo. A este fenómeno se agrega que en junio de 2020 "el $44 \%$ de los países de la región no alcanzaba la velocidad de descarga que permite desarrollar varias actividades en línea simultáneamente" ( $p$. 4), aunado a la limitación en el acceso a Internet y el uso de aplicaciones debido al alto costo del servicio de banda ancha móvil y fija; por ejemplo, para la población del primer quintil de ingresos llegaba al $14 \%$ y el $12 \%$, respectivamente.

Con relación a este aspecto, del total de servicios con que cuentan los docentes encuestados para enseñar destacan la luz eléctrica (93.2\%) y la señal de celular (86.5\%), apenas tres de cada cinco disponen de Internet (59.4\%), la mayoría tienen televisión (74.4\%) y una tercera parte, radio (31.6\%). En contraste, los alumnos muestran una ostensible falta de servicios en comparación con sus maestros: el 75.2\% tienen luz eléctrica, el $10.5 \%$ está provisto de Internet, el $51.1 \%$ de señal de televisión y el $53.4 \%$ de celular, mientras que los datos acerca de la radio son similares (34.6\%). 
Tabla 3. Porcentaje de disponibilidad de servicios y equipamiento tecnológico. Sierra Tarahumara, Chihuahua

\begin{tabular}{lccccc}
\hline \multicolumn{1}{c}{ Municipios } & Computadora & $\begin{array}{c}\text { Teléfono } \\
\text { fijo }\end{array}$ & $\begin{array}{c}\text { Teléfono } \\
\text { celular }\end{array}$ & Internet & TV de paga \\
\hline Batopilas & 3.1 & 3.1 & 26.8 & 0.8 & 15.4 \\
\hline Carichí & 10.4 & 11.4 & 56.4 & 17.0 & 35.7 \\
\hline Urique & 7.3 & 3.5 & 50.7 & 3.9 & 30.5 \\
\hline Guachochi & 14.6 & 6.9 & 59.5 & 9.5 & 23.5 \\
\hline Guadalupe & 7.8 & 4.4 & 56.5 & 8.9 & 39.5 \\
\hline Morelos & 4.9 & 1.6 & 40.2 & 4.2 & 36.2 \\
\hline Maguarichi & 6.2 & 0.2 & 57.1 & 1.1 & 41.1 \\
\hline Uruachi & 4.9 & 2.7 & 42.4 & 0.5 & 27.1 \\
\hline Guazapares & 13.1 & 6.9 & 70.7 & 9.0 & 49.5 \\
\hline Bocoyna & 15.3 & 6.9 & 71.5 & 12.2 & 43.8 \\
\hline Chínipas & 12.2 & 11.1 & 53.4 & 9.5 & 61.1 \\
\hline Promedio & 9.4 & 5.3 & 53.4 & 6.9 & 36.7 \\
\hline Estado & 42.7 & 40.2 & 91.8 & 56.8 & 42.1 \\
\hline
\end{tabular}

Fuente: elaboración propia a partir de INEGI (2021).

En la Tabla 3 se aprecia que la diferencia entre el promedio de computadoras en la Sierra Tarahumara y el porcentaje del Estado de Chihuahua es de 33.3 puntos, para el teléfono fijo de 34.8, aumenta con el celular a 38.4, se incrementa hasta 49.9 en la conexión a Internet y se aproxima a 5.4 puntos con la televisión de paga.

La discordancia entre las cifras estatales y los 11 municipios de la Sierra donde trabajan los maestros que participaron en la investigación, así como la que se produce con estudiantes y maestros repercute de forma directa en las interacciones que se establecen durante la contingencia sanitaria: los docentes cubren el $71.4 \%$ de los servicios básicos y sus alumnos alcanzan el 56.2\%. La desviación de 15.2 puntos provoca un desequilibrio en las posibilidades de comunicación, que se multiplica con la dispersión geográfica y las distancias que hay entre los lugares de residencia de estudiantes y maestros. Como consecuencia, los recursos que utilizan los docentes se concentran en los cuadernillos de trabajo (92.5\%), los contactos por WhatsApp (55.6\%), los libros de texto (51.9\%) y en menor medida, los complementos didácticos (40.6\%). En un segundo nivel se ubican las clases por televisión (30.8\%) y de modo testimonial, la plataforma Classroom (6\%), las llamadas telefónicas $(0.8 \%)$ y los mensajes de texto (0.8\%). La carencia de soportes tecnológicos coincide con el empleo abrumador de los materiales impresos, sea en forma de cuadernillos o libros de texto, una constatación de que los medios electrónicos son "insuficientes para los retos que supone adecuar la oferta y los formatos pedagógicos a estudiantes en entornos desfavorecidos" (CEPAL-UNESCO, 2020, p. 10). 
De esta suerte, el recurso "Aprende en casa" que promueve la SEP a través de diferentes televisoras resulta funcional para un limitado número de docentes (3.9\%). La mayoría valoran que ha sido nada (11.5\%) o poco funcional $(59.2 \%)$ porque los contenidos están descontextualizados, solo algunos alumnos ven los programas e incluso recalcan que no hay luz eléctrica o los canales no llegan a las comunidades donde viven los estudiantes. Estos porcentajes son esclarecedores sobre la efectividad de las propuestas que las autoridades educativas pretenden hacer válidas en un contexto como el de la Sierra Tarahumara. Por el contrario, en alusión a una encuesta aplicada a 291279 docentes, directores, supervisores, asesores técnicos pedagógicos y otras figuras educativas, la SEP recalca que más del $86 \%$ otorgaron una calificación de 8 a 10 a los programas de "Aprende en Casa II" y que el 47.2\% de los maestros lo usan para dar continuidad al aprendizaje de sus alumnos (SEP, 2021, 10 de enero). Evidentemente, muchos docentes no pueden estar más que en desacuerdo:

No se trabaja con clases de televisión, no funcionan y luego es televisión de paga. No está parejo el piso para todos. Cómo van a poner los padres de familia SKY si tienen otras prioridades económicas. Las clases de preescolar se emiten a las siete de la mañana y son aburridas (E7-PRMG).

No es operativo, es cero factible. En la comunidad no hay Internet, no hay televisión, no hay luz. No se puede trabajar de esa forma (E3-PMI).

No es de extrañar que en una región con apenas infraestructura tecnológica los modelos de aprendizaje a distancia sin conexión representen la mejor y única opción (Banco Mundial, 2020) e incluyan el diseño y distribución de materiales impresos, el llenado de los libros de texto, además de la diversificación de los medios, formatos y plataformas (CEPAL-UNESCO, 2020).

Aquí como estrategia solo utilizamos los cuadernillos. Los cuadernillos los hacemos nosotras, las tres maestras de segundo. La mayoría son actividades del libro de texto. Los papás se cooperan para tinta de impresión y para los niños con necesidades educativas los adaptamos (E2-PCG).

Los cuadernillos nosotros los pagamos de nuestra bolsa. Cada tres semanas vamos a la comunidad a entregar cuadernillos y a revisar los que dejamos (E4-PMG).

En los cuadernillos de trabajo hay una tendencia muy marcada hacia las dificultades que representa enseñar y aprender desde casa. La tercera parte de los docentes confirman que los estudiantes realizan las actividades, pero mal resueltas (36.9\%) y uno de cada cuatro reconocen que muy pocos contestan todos los ejercicios (29.5\%). El resto aseguran que los alumnos regresan las actividades de forma correcta y que este material es eficaz para aprender (33.6\%). En este apartado se debe precisar que, dadas las características que tiene la educación a distancia en una zona tan dispersa geográficamente, en algunos casos las tareas no son revisadas y a veces, ni siquiera vistas por sus maestros.

El mayor problema con los cuadernillos en este contexto es que la gente tiene que salir a trabajar, los recogen en la tienda y a muchos niños no les llegan. Como no hay clases presenciales se van a trabajar porque no tienen necesidad de asistir a la escuela; por eso tenemos niños con calificación pendiente (E8-PMI). 
Por tanto, la relación entre las posibilidades tecnológicas y los déficits en la comunicación obliga a que las propuestas de aprendizaje sean multimodales y adecuadas al contexto (Banco Mundial, 2020). Apenas el $12.7 \%$ de los maestros encuestados afirman no tener dificultades para comunicarse con los alumnos y, por contra, nueve de cada diez manifiestan que en las comunidades donde viven los estudiantes no hay conectividad a Internet (35.1\%) ni señal de celular (23.7\%), a lo que se suma que no hay luz eléctrica y tampoco televisión (9.2\%). El resto de las dificultades (18.3\%) se reparten en una gama de situaciones que se asocian entre sí: no se pueden utilizar plataformas virtuales, no todos tienen celular propio, solo usan datos para navegar en Internet, no disponen de saldo, en el hogar hay más de dos hijos y un solo teléfono inteligente, etc. Esta clase de eventualidades explican por qué en ocasiones los docentes recurren a los medios que están a su alcance:

Solicito que el gobernador indígena cite a los alumnos. Solo así se pueden juntar a los niños porque viven muy lejos. No hay teléfono ni correo electrónico. Es necesario estar en la escuela de manera presencial uno o dos días por quincena para platicar con los padres y estudiantes. Así le hicimos el año pasado, ahora estamos de manera presencial (E6-TSMI).

No sorprende que casi la mitad de los maestros consideren que las estrategias de la para atender la pandemia no son apropiadas porque no se adaptan al contexto de los alumnos (48.1\%) o que una proporción similar las valore como más o menos apropiadas (44.3\%). En ambos extremos se sitúan un grupo reducido de docentes que las juzgan como apropiadas y útiles (3.8\%), mientras que la misma cantidad recurre a otras alternativas y no atienden las sugerencias de las autoridades (3.8\%). Así pues, en las zonas rurales de la Sierra Tarahumara quedan inhabilitadas las soluciones digitales.

\subsection{Atención a los estudiantes}

La implementación del aprendizaje a distancia supone una mayor carga para los padres de familia que ayudan a sus hijos en las actividades que encargan los maestros o se transmiten por televisión. Las personas que apoyan a los alumnos para hacer las tareas son diversas: la mayoría afirman recibir el acompañamiento de las mamás (91.6\%) y una proporción menor recae en los papás (49.6\%) o hermanos (48.1\%). En tercer lugar, se sitúan los abuelos (19.8\%) y uno de cada diez alumnos no tienen ayuda (10.7\%). Los compañeros, los tíos, tutores o vecinos apenas son mencionados (0.8\% por categoría).

La capacitación de los padres es un elemento crítico, ya que la educación desde los hogares es una novedad en este tipo de contexto y significa no solo un alto desgaste emocional sino una inversión de tiempo que repercute en sus actividades laborales (Banco Mundial, 2020). Así lo relata una docente de educación preescolar: "A los padres de familia se les dio un paquete muy grande. Existe mucho cansancio. El tiempo es limitado, tienen otros hijos y trabajan, no es tan fácil" (E5-PRMI).

La brecha en la equidad educativa se observa en la disposición de hardware, software y conectividad, además de las dificultades que tienen las familias con respecto a las habilidades digitales, principalmente cuando se atiende a estudiantes de preescolar y del primer ciclo de educación primaria. Esta problemática coincide con la aclaración que hace la CEPAL-UNESCO (2020): 
No se trata solo de una diferencia de acceso a equipamiento, sino también del conjunto de habilidades que se requieren para poder aprovechar esta oportunidad, que son desiguales entre estudiantes, docentes y familiares a cargo del cuidado y la mediación de este proceso de aprendizaje que hoy se realiza en el hogar (p. 7).

Las habilidades que los docentes de la muestra identifican en los padres de familia para usar la tecnología son muy limitadas. Más de la mitad saben cómo enviar mensajes por WhatsApp (61.1\%), pero el porcentaje se reduce cuando se trata de tomar y enviar fotografías $(44.2 \%)$, unos cuantos pueden gestionar llamadas para trabajar en equipo (10.7\%), disminuyen los que saben manejar archivos .pdf, .ppt o videos $(6.9 \%)$ y un buen número no utilizan WhatsApp por diferentes motivos (38.2\%).

A falta de un acompañamiento oportuno y efectivo de los padres de familia, la alternativa es el apoyo pedagógico de los maestros. Casi todos los docentes retroalimentan las tareas de forma personalizada, con sugerencias para corregir los trabajos (84.7\%), otros mandan mensajes a los grupos de WhatsApp y señalan los aspectos incorrectos (4.6\%), y la tercera parte escriben notas para felicitar y animar (33.6\%). Casi ningún maestro afirma que no retroalimenta (3.1\%).

Organizamos reuniones con los niños y padres de familia para hacer recomendaciones. De 18 padres, solo tres saben leer y escribir. Sí, hay voluntad, pero no los pueden apoyar. Los niños tampoco tienen dónde investigar las tareas, tienen muy pocos recursos y además deben trabajar (E3-PMI).

En los cuadernillos se hacen observaciones, a sabiendas de que a lo mejor no van a llegar al alumno. Igualmente, muchos padres no saben leer (E4-PMG).

Por estas y otras causas, el compromiso profesional lleva a que los docentes pongan en riesgo su salud y la de los alumnos e infrinjan las reglas de distanciamiento:

A partir de enero las actividades son de manera presencial, un día para los niños que vienen de fuera y otro día para los niños de la comunidad. La estrategia consiste en tomar las medidas necesarias porque a distancia no se puede y de la otra manera no se obtiene aprendizaje. Los cuadernillos de la Secretaría de Educación del Estado de Chihuahua no funcionan porque están muy elevados. Los adaptamos, pero la mayoría de los padres son analfabetas (E12-TSI).

\subsection{Funciones docentes}

Una de las consecuencias de la pandemia ha sido la saturación e intensificación de la práctica docente: el 72.6\% ocupan 4, 5 o más horas a la semana para planear las clases a distancia y el resto, 3 (19.8\%) o 2 horas (7.6\%). Asimismo, destinan entre 1 y 5 horas semana (21.8\%) para atender a sus alumnos, aunque casi la mitad emplean entre 6 y 10 horas (43.6\%). De 11 a 15 horas (12\%) y de 16 a 20 horas (12.8\%) congrega a uno de cada cuatro docentes (24.8\%), mientras que un número menor dedica más de 20 horas (9.8\%). Esta distribución se puede comparar con las 22.5 horas clase semana en la modalidad presencial que se plantean para la educación primaria, las 35 de secundaria y las 15 de preescolar (Acuerdo número 01/01/20).

El tiempo que los maestros utilizan para evaluar está más disgregado que el de la planeación. Más de la mitad ocupan 4, 5 o más horas (57.2\%), un porcentaje menor 3 horas (22.9\%) y apenas uno de cada cinco le asignan 1 o 2 horas semana (19.9\%). Llama la atención que, aunque la currícula propone determinados periodos 
lectivos en la variante presencial, el $65.4 \%$ de los docentes brindan a la enseñanza menos de 10 horas en la modalidad a distancia porque priorizan la aplicación de tareas. Asimismo, el 57\% destina de 4 a 5 horas a evaluar, pero la mayor proporción del tiempo revisan trabajos e informan acerca de los errores que cometieron los alumnos.

La tercera parte de los encuestados (31\%) reúne a un grupo de docentes que no se han visto sobrepasados por la carga de trabajo que representa la educación a distancia. En un segundo nivel se sitúan uno de cada cinco maestros, todos aquellos que invierten la misma cantidad de horas a las tres actividades que caracterizan su desempeño (22.5\%). Por último, casi la mitad de los docentes han sufrido una sobrecarga (46.3\%), especialmente respecto a la planeación y evaluación de la enseñanza.

Dedico mucho tiempo a planear y diseñar actividades. Me centro más en que las actividades sean entendibles, fáciles para los padres de familia y el estudiante. Dejo un poco de lado el aprendizaje esperado (E1-SCG).

Para empezar, no sabíamos planear a distancia, pensábamos que era lo mismo. Es mucho tiempo de revisión, ha aumentado exageradamente el trabajo en casa y más, porque tenemos que estar también con los hijos que van a la escuela (E10-PMG).

\subsection{Formación continua}

Los programas de formación docente y para directivos para optimizar la educación a distancia deberían ir encaminados a mejorar el uso de las tecnologías, así como a realizar adaptaciones con la intención de reducir la brecha digital y de aprendizaje en las zonas más desfavorecidas. En el cuestionario, los maestros señalan que el tipo de apoyo que desearían recibir para enseñar con mayor eficacia se refiere a la adquisición de estrategias didácticas (67.2\%), involucrar a los padres de familia (53.4\%), evaluar (52\%) y planear la enseñanza (50.4\%), cuatro necesidades que son un reflejo de las carencias que detectan en su quehacer diario, las habilidades digitales que tienen para enseñar a distancia y la escasa capacitación que han recibido de las autoridades educativas.

A este conjunto de contenidos se podrían incorporar la formación para determinar los retrasos en el aprendizaje, ajustar la enseñanza a las características de los alumnos o identificar las áreas prioritarias del currículo (Banco Mundial, 2020), además de las 18 competencias para integrar la tecnología a la educación, entre las que destacan: "elegir adecuadamente las TIC en apoyo a metodologías específicas de enseñanza y aprendizaje; organizar el entorno físico de modo tal que la tecnología sirva para distintas metodologías de aprendizaje de manera inclusiva" (UNESCO, 2019 , p. 6). Aunque antes habría que solucionar los problemas de conectividad que se enfrentan en el medio rural: "Mandan evidencias por Internet, pero algunos videos son muy pesados y se batalla para poder verlos" (E2-PCG).

\subsection{Desempeño y deterioro profesional}

En general, predomina la tendencia negativa sobre si se lograron o no los aprendizajes esperados que marcan los programas de estudio. Aproximadamente, uno de cada tres maestros afirma que se alcanzaron el $50 \%$ de los propósitos (36.6\%) y uno de cada cuatro sostiene que apenas el $25 \%$ (22.9\%), mientras que una proporción menor garantiza que se llegó hasta el 75\% (37.4\%). Los demás no están 
seguros (1\%) o creen que se obtuvo el $100 \%$ de los aprendizajes esperados (2.1\%). Estas percepciones se vinculan con las deficiencias que encuentran en la educación a distancia (59.5\%).

En una reunión de supervisores de cuatro sectores ubicados en la Sierra Tarahumara que agrupan a 289 escuelas y 684 docentes, celebrada en noviembre de 2020 para hacer un diagnóstico del grado de afectación en los aprendizajes esperados, se presentaron datos muy similares: un $16 \%$ aseguraron que los alumnos alcanzaron a dominar todos o casi todos los aprendizajes esperados (entre $80 \%$ y $100 \%$ ), el $38.6 \%$ afirmó que más de la mitad y el 31\% que apenas se lograron dos de cada cinco aprendizajes esperados. El 11.8\% concluyó que una quinta parte, dos docentes más dijeron que ningún aprendizaje y el 1.9\% no contestaron. Estos porcentajes son consecuencia, entre otros factores, de las disposiciones que la SEP emitió para la educación básica en el cierre del ciclo escolar 2019-2020 y en diciembre de 2020.

En caso de no tener elementos adicionales para la valoración del tercer periodo [la calificación] será el promedio de las calificaciones obtenidas en los dos periodos de evaluación previos; considerando criterios de equidad y el interés superior de niñas, niños y adolescentes; se procurará su continuidad al siguiente grado escolar o nivel educativo (Acuerdo número 12/06/20, p. 4).

En caso de que así lo determine el docente, y al no existir condiciones objetivas para formular un criterio, las calificaciones o valoraciones que se registren serán preliminares; las calificaciones definitivas se registrarán al final del ciclo escolar 2020-2021 (Acuerdo número 26/12/20, p. 3).

La terapia de la apariencia. La continuidad a la que se refieren ambos acuerdos significa evitar la reprobación y se sustenta en el siguiente argumento: "La información obtenida mediante la evaluación es la base para identificar y modificar aquellos aspectos del proceso que obstaculizan el logro de los propósitos educativos; ello implica pasar a segundo término su papel en la asignación de calificaciones" (Acuerdo número 12/06/20, p. 5).

La terapia de la legitimación. En una encuesta aplicada por la Comisión Nacional para la Mejora Continua de la Educación (MEJOREDU, 2020), cuyo objetivo era conocer las experiencias educativas durante el confinamiento y que se aplicó a 34990 estudiantes de educación básica, se menciona "que valoran la retroalimentación cercana y frecuente de sus docentes, disfrutan asumir un papel más activo y autónomo en su aprendizaje y gozan la convivencia con sus pares" (p. 46). A estas opiniones se añade que los alumnos reforzaron sus aprendizajes previos (51.8\%), adquirieron nuevos conocimientos sobre sus materias (47.8\%), aprendieron a hacer cosas nuevas relativas a aprendizajes extraescolares (50.5\%) y a usar aplicaciones o plataformas (48.2\%).

La terapia de la evidencia. En un plano opuesto se encuentra la realidad de la Sierra Tarahumara. Un director con grupo de una telesecundaria bidocente indígena se lamenta de que "los alumnos no cuentan con el nivel académico para el que están cursando, no saben leer, escribir, realizar operaciones matemáticas. No responden el cuadernillo como tal"; además, asevera que no puede reprobar "por indicaciones de 
las autoridades" y que "evaluamos el desarrollo de los estudiantes, en cuanto al inicio de la pandemia, cómo estaba el alumno y cómo está ahora", y que por eso "no hay evaluación, solo se simula porque no hay con qué evaluar" (E6-TSMI).

La terapia de la benevolencia. En la normativa oficial también se solicita reconocer el esfuerzo de los alumnos y sus familias, motivar a quienes se comunican esporádicamente y se deja abierta "la posibilidad de recuperar a quienes, hasta el momento, no establecen comunicación" (Acuerdo número 12/06/20, p. 2).

El ciclo escolar 2020-2021 iniciará el 10 de agosto de 2020 con una etapa remedial o de nivelación, un periodo de valoración diagnóstica y de trabajo docente para resarcir rezagos e insuficiencias en el aprendizaje correspondiente al grado anterior y tendrá una duración mínima de tres semanas; el colectivo docente o el titular del grupo podrán determinar la ampliación de este periodo (p. 5).

Al parecer, estas medidas han sido bastante positivas. En el concentrado de calificaciones del primer periodo de evaluación del ciclo escolar 2020-2021 de una zona escolar de educación primaria situada en una de las regiones más marginadas de la Sierra Tarahumara, que reúne a 11 escuelas multigrado y a 359 estudiantes, los promedios por grado oscilaron entre el 8.3 de sexto al 7.6 de primer año (alumnos a quienes los docentes no han tenido oportunidad de conocer), para alcanzar una media general de 8 . Desde luego, sin reprobados.

Lejos de que la educación a distancia haya revalidado la función formativa de la evaluación, ya que "a través de ejercicios de diagnóstico y de seguimiento, permite a las y los docentes proporcionar retroalimentación a sus estudiantes y modificar sus estrategias pedagógicas para que sean más efectivas" (CEPAL-UNESCO, 2020, p. 9 ), las valoraciones de los docentes prueban que las calificaciones son un velo que cubre las pérdidas de aprendizaje:

Yo creo que no se lograron los aprendizajes esperados porque a los niños ya se les olvidaron, por eso estoy volviendo a empezar. De por sí hay mucha movilidad en las comunidades indígenas. Si estábamos atrasados en comparación con otras escuelas, ahora virtual, imagínese (E8-PMI).

No se lograron los aprendizajes, no se necesitan rúbricas para darse cuenta de eso. A los niños ya se les olvidaron muchas de las cosas que aprendieron de manera presencial (E5-PRMI).

La consulta de los resultados de Plan Nacional para la Evaluación de los Aprendizajes (PLANEA) demuestra que ya en condiciones normales la pobreza de aprendizaje era muy común en México:

- En 2018, el 49\% de los estudiantes que egresaron de sexto de primaria alcanzaron en Lenguaje y comunicación el nivel I de desempeño (insuficiente); 33\% logró el nivel II (básico); 15\% el nivel III (satisfactorio), y solo 3\% obtuvo el nivel IV (sobresaliente). En Matemáticas, el 59\% de los alumnos se encontraba ubicado en el nivel I; $18 \%$ en el nivel II; $15 \%$ en el nivel III; y sólo $8 \%$ en el nivel IV.

- En 2017, la evaluación de tercero de secundaria mostró que el 34\% de los alumnos alcanzaron el nivel I; $40 \%$ el nivel II; $18 \%$ el nivel III; y $8 \%$ el nivel IV. En Matemáticas el $65 \%$ obtuvieron el nivel I; $22 \%$ el nivel II; $9 \%$ el nivel III; y $5 \%$ el nivel IV (INEE, 2019). 
En el mismo informe, el Instituto Nacional para la Evaluación de la Educación (INEE) especifica que en las escuelas indígenas y comunitarias "de cada 10 estudiantes, entre 2 y 3 superan el nivel I de logro, tanto en Matemáticas como en Lenguaje y comunicación" (p. 100). En definitiva, si en ese entonces el sistema educativo no había sido capaz de revertir la tendencia nacional respecto al aprendizaje, en estos momentos la inequidad que sufren las comunidades rurales vulnera todavía más el derecho a recibir una educación de calidad.

Finalmente, casi todos los maestros consideran que la educación a distancia les ha provocado más estrés que las clases presenciales (92.4\%), muy pocos se refieren a que sienten la misma presión (6.6\%) y solo un docente de 133 afirma que con esta modalidad está más relajado. Estos porcentajes son concluyentes y se relacionan con las dificultades identificadas en el cuestionario y las entrevistas. Paradójicamente, la mayor autonomía para tomar decisiones y enfocarse en las áreas básicas del currículum se ha convertido en desgaste e insatisfacción profesional. La salud mental, la capacidad de adaptación y la resiliencia emocional adoptan las mismas expresiones:

Ahora en la pandemia es menos el tiempo que trabajo porque la estrategia del gobierno no funciona para el nivel de telesecundaria, pero se me hace más estresante porque hay menos resultados y nulo apoyo de los padres de familia (E12-TSI).

La parte más estresante es saber que no están aprendiendo, que el esfuerzo realizado no es suficiente. Además, a esto de la pandemia no se le ve la orilla, no se sabe cuándo vamos a regresar a presencial (E5-PRMI).

El estrés está cuando me esmero y la gente no cumple, porque hay muchos factores que influyen y están fuera de mis manos. En el salón yo puedo convencer a mis niños para que trabajen, pero ahora tengo que convencer primero a los papás porque son niños de segundo (E11-PCG).

El Banco Mundial (2018) sostiene que las personas que se encuentran en desventaja debido a su nivel económico, ubicación geográfica, etnia, género o discapacidad son quienes menos aprenden. Como un factor yuxtapuesto, la pandemia por la COVID-19 ha ampliado las brechas educativas y por derivada, una inequidad que se refleja en la pobreza del aprendizaje y que, como anticipan los docentes, se va a agravar con el tiempo.

\section{Discusión y reflexiones}

Las políticas propuestas por diferentes organismos internacionales (Banco Mundial 2020; UNESCO, 2020) para ayudar a los sistemas educativos a afrontar los impactos provocados por el cierre de escuelas tiene como uno de sus objetivos aminorar la pérdida de aprendizajes. Entre las recomendaciones, se plantea prevenir la deserción escolar mediante la comunicación y el apoyo financiero a los estudiantes, usar la tecnología de manera inclusiva para que no se descuide a quienes tienen menores niveles de acceso, crear inventarios de contenidos básicos y emplear material de estudio que se adecúe a las características de los alumnos. Sin embargo, en el contexto de la Sierra Tarahumara muchas estrategias no han sido efectivas porque la mayoría de las familias no están en posición de apoyar el aprendizaje de 
sus hijos, las dificultades para mantener la comunicación son irreversibles y ninguna de las soluciones adoptadas han podido reemplazar la experiencia presencial, de ahí que muchos docentes hayan decidido retomar el contacto directo con los estudiantes.

Ciertamente, la pandemia ha coartado la autonomía de los docentes, de los alumnos y de los padres de familia porque se han visto abocados a una vorágine sin sentido formativo que se puede cifrar en un aumento de la deserción cuando se regrese a la normalidad. En la situación actual no basta con querer recuperar el aprendizaje y el tiempo porque la desatención educativa en la Sierra Tarahumara tiene carácter endémico y más, cuando una de las consecuencias de la educación a distancia va a ser el incremento de la heterogeneidad en las aulas, así como la convicción de que las prácticas de enseñanza van a ser menos eficaces (Banco Mundial, 2020). Tampoco será suficiente enfocarse en la enseñanza de la lectura, la escritura y las operaciones básicas como contenidos fundamentales porque la simplificación del currículum ahonda en las diferencias de partida.

La crisis de aprendizaje y la imposibilidad de cumplir con los objetivos de la Agenda 2030 nos obliga a preguntar por qué seguimos inmersos en la misma dinámica y a reflexionar si todavía la finalidad de la educación es garantizar el aprendizaje de todos los alumnos. Por esta razón, el dilema entre poner una pausa o dar continuidad a la educación escolar se ha de resolver desde la óptica de la equidad y la inclusión. La disyuntiva entre priorizar la seguridad y disminuir el riesgo de contagio o reanudar el proceso de recuperación del aprendizaje debe ser producto de un convenio que incumba a los padres de familia, estudiantes y maestros.

La decisión primordial que se debe tomar después de la COVID-19 es si comenzar el próximo ciclo escolar como si nada hubiera ocurrido o evaluar el nivel de aprendizaje del alumnado para reestructurar la secuencia grado-nivel, un acuerdo que también corresponde a los principales actores educativos. En esta contextura, la igualdad educativa, si cabe, tendrá que cobrar mayor relevancia en la agenda pública cuando a nivel mundial se supere la pandemia.

\section{Referencias}

Acuerdo número 01/01/20 por el que se emiten los Lineamientos de ajuste a las horas lectivas señaladas en el diverso número 592 por el que se establece la Articulación de la Educación Básica, para los ciclos escolares 2019-2020 y 2020-2021. Diario Oficial de la Federación, México, 23 de enero de 2020.

Acuerdo número 12/06/20 por el que se establecen diversas disposiciones para evaluar el ciclo escolar 2019-2020 y cumplir con los planes y programas de estudio de Educación Básica (preescolar, primaria y secundaria), Normal y demás para la formación de maestros de Educación Básica aplicables a toda la República, al igual que aquellos planes y programas de estudio del tipo Medio Superior que la Secretaría de Educación Pública haya emitido, en beneficio de los educandos. Diario Oficial de la Federación, México, 5 de junio de 2020.

Acuerdo número 26/12/20 por el que se establecen las orientaciones pedagógicas y los criterios para la evaluación del aprendizaje para la educación preescolar, primaria y secundaria en el periodo de contingencia sanitaria generada por el virus SARS-CoV2 (COVID-19) para el ciclo escolar 2020-2021. Diario Oficial de la Federación, México, 28 de diciembre de 2020.

Banco Mundial (2018). Informe sobre el desarrollo mundial 2018: Aprender para hacer realidad la promesa de la educación. Washington, DC: Banco Mundial. 
Banco Mundial (2019). Ending Learning Poverty: What Will It Take? Washington, DC: Banco Mundial. Banco Mundial (2020). COVID-19: Impacto en la educación y respuestas de política pública. Washington, DC: Grupo Banco Mundial Educación.

Banco Mundial (2021). World Bank Education COVID-19 School Closures Map. Recuperado de https://bit.ly/2SyqWvJ

Baptista, P., Almazán, A., Loeza, C., López, V. y Cárdenas, J. (2020). Encuesta Nacional a Docentes ante el COVID-19. Retos para la educación a distancia. Revista Latinoamericana de Estudios Educativos, 50(núm. esp.), 41-88.

CEPAL-UNESCO (2020, agosto). La educación en tiempos de la pandemia de COVID-19. Informe COVID-19. Recuperado de https://bit.ly/3hZeW19

CEPAL-ONU (2020, 26 de agosto). Universalizar el acceso a las tecnologías digitales para enfrentar los efectos del COVID-19. Informe Especial COVID-19 n 7. Recuperado de https://bit. ly/3fUvq7M

(COESPO) (2017). Consejo Estatal de Población. Programa estatal de población 2017-2021. Chihuahua: Gobierno del Estado de Chihuahua.

Creswell, J. (2003). Diseño de investigación. Enfoques cualitativo, cuantitativo y con métodos mixtos. Thousand Oaks: California Sage Publications.

Johnson, B. y Onwuegbuzie, A. (2004). Mixed Methods Research: A Research Paradigm Whose Time Has Come. American Educational Researcher Association, 33(7), 14-26.

IIPE-UNESCO (2020). Instituto Internacional de Planeamiento de la Educación. Sistematización de respuestas de los sistemas educativos de América Latina a la crisis de la COVID-19. Recuperado de https://bit.ly/3vyyRle

INEE (2019). Instituto Nacional para la Evaluación de la Educación. La educación obligatoria en México. Informe 2019. México: INEE.

INEGI (2021). Instituto Nacional de Estadística y Geografía. Panorama sociodemográfico de Chihuahua: Censo de Población y Vivienda 2020. México: INEGI.

INEGI y SEP (2014). Instituto Nacional de Estadística y Geografía y Secretaría de Educación Pública. Tabulados del Censo de Escuelas, Maestros y Alumnos de Educación Básica y Especial, CEMABE 2013. México: INEGI y SEP.

MEJOREDU (2020).Comisión Nacional para la Mejora Continua de la Educación. Experiencias de las comunidades educativas durante la contingencia sanitaria por covid-19. Educación básica. México: MEJOREDU.

Navarrete, Z, Manzanilla, H. y Ocaña, L. (2020). Políticas implementadas por el gobierno mexicano frente al COVID-19. El caso de la educación básica. Revista Latinoamericana de Estudios Educativos, 50(núm. esp.), pp. 143-172.

SEP (2021, 10 de enero). Secretaría de Educación Pública. Boletín SEP no. 7. Regresan a clases más de 25 millones de alumnos de Educación Básica. Recuperado de https://bit.ly/3usHeUh

UNESCO. Organización de las Naciones Unidas para la Educación, la Ciencia y la Cultura (2017). Declaración de Buenos Aires Reunión Regional de Ministros de Educación de América Latina y el Caribe. Santiago de Chile: Oficina Regional de Educación para América Latina y el Caribe (OREAL).

UNESCO (2019). Marco de competencias de los docentes en materia de TIC elaborado por la UNESCO. París: UNESCO.

UNESCO (2020). COVID-19 Educational Disruption and Response. Recuperado de https://bit. Iy/3g6PtAn. 